\title{
Cardiovascular magnetic resonance imaging and its role in the investigation of stroke: an update
}

\author{
Ana Catarina Fonseca ${ }^{1}\left[\right.$ ] José M. Ferro ${ }^{1} \cdot$ Ana G. Almeida $^{2}$
}

Received: 7 December 2020 / Revised: 29 December 2020 / Accepted: 31 December 2020

(c) Springer-Verlag GmbH, DE part of Springer Nature 2021

\begin{abstract}
Recent advances in complementary diagnostic exams have helped to clarify stroke etiology, not only by helping to confirm established stroke causes but also by unveiling new possible stroke mechanisms. Etiological investigation for cardioembolic stroke has benefited in the last years from information provided by studies analysing serum biomarkers, heart rhythm monitoring and imaging methods like cardiovascular magnetic resonance (CMR) imaging. CMR has been particularly important for the characterization of possible new cardioembolic stroke mechanisms including atrial cardiomyopathy, silent myocardial infarction and cardiomyopathies.
\end{abstract}

Keywords Stroke $\cdot$ Etiology $\cdot$ Cardiac magnetic resonance $\cdot$ Cryptogenic $\cdot$ Cardioembolism $\cdot$ Atrial cardiomyopathy

\section{Introduction}

The classification of ischaemic stroke subtypes used, in general, the simple and reliable TOAST typology [1], which, however, leaves around a $1 / 4$ of the cases unclassified. More detailed systems of classification, such as the ASCOD [2], decrease this proportion of cryptogenic strokes, by attributing the cause of stroke to "minor" arterial or cardiac embolic sources [3]. ASCOD is unfortunately too complex to use in daily practice. A related difficulty is the operational definition of cryptogenic stroke. A review of 16 clinical practice guidelines and 7 consensus statements found that none of the reports provided guidance on the extent of investigation needed to classify a stroke as cryptogenic [4].

Stroke may be labelled unclassified in patients with multiple causes or those with incomplete or delayed investigations. In the remaining strokes of unknown cause, most are embolic with an unknown source (ESUS) [5]. In some of these patients, paroxysmal atrial fibrillation (AF) or a

Ana Catarina Fonseca

acfonseca@medicina.ulisboa.pt

1 Department of Neurology, Institute of Molecular Medicine, Hospital de Santa Maria, University of Lisboa, Avenida Professor Egas Moniz, 1649-035 Lisbon, Portugal

2 Department of Cardiology, Hospital de Santa Maria, CCUL, University of Lisboa, Lisbon, Portugal "minor" cardiopathy will be identified. Carotid webs [6-8] and cancer are also other possible sources of ESUS.

Advances in vessel imaging have unveiled other potential causative mechanisms including non-stenotic carotid or vertebral atheroma, ostium or branch intracranial artery and aortic arch atheroma. In the NAVIGATE ESUS trial, involving 7213 patients ESUS patients, the 3 most prevalent potential sources of embolism were atrial cardiopathy (37\%), left ventricular disease (36\%), and arterial atherosclerosis (29\%) [9], but 7.5\% had cancer [10]. Among participants who had transesophageal echocardiography (TEE), 29\% had aortic arch atheroma [11]. Among those with intracranial arterial imaging, $16 \%$ had intracranial atherosclerosis [12], while $12.6 \%$ patients met the criteria for intracranial branch atheroma [13]. In other recent studies, between 23.6 and $38.6 \%$ of ESUS patients had ipsilateral $<50 \%$ carotid plaques $[14,15]$. Nonstenotic carotid plaques were significantly more common on the side of the ischemic stroke [16]. They were also ipsilateral to the infarction in patients with ESUS, but not in patients with AF, supporting an underlying atheroembolic mechanism in a subset of ESUS patients [17]. The identification and quantification by MRI of certain carotid plaque characteristics, including lipid-rich necrotic core, thin/ruptured fibrous cap, and intraplaque hemorrhage (IPH), may improve the prediction of future stroke among ESUS patients [18]. In a systematic review of 7 studies with 354 ESUS patients, the prevalence of IPH ipsilateral to the 
ischemic lesion was $25.8 \%$. The odds of having IPH on the ipsilateral side versus the contralateral side was 6.92 [19].

The carotid web is another cause of cryptogenic stroke, particularly in younger patients without vascular risk factors $[6-8,20]$. The web is a thin intraluminal filling defect along the posterior wall of the carotid bulb, on oblique sagittal section of CT angiography (CTA), seen as a septum on axial CTA. Pathologically, it is an intimal fibromuscular dysplasia. The web causes flow stagnation and remote embolization of fibrin-based clots. Carotid webs were found at a significantly higher frequency in patients with cryptogenic ischemic strokes compared to controls. Additionally, intracranial large vessel occlusions were more common in patients with symptomatic carotid webs, presenting with ipsilateral strokes [21].

These studies demonstrate the impact that recent advances in complementary diagnostic exams have had in helping to clarify stroke etiology. Not only by aiding to confirm stroke etiology but also by unveiling new possible stroke mechanisms. Cardioembolic stroke investigation has benefited in the last years from information provided by studies analysing serum biomarkers, heart rhythm monitoring and imaging exams like cardiovascular magnetic resonance (CMR) imaging [22, 23]. CMR has had a progressively more important role in the evaluation of patients suspected of having cardioembolic stroke.

\section{Cardiovascular magnetic resonance}

CMR is an imaging technique that is complementary to other modalities such as echocardiography, cardiac CT and nuclear cardiology. This technique is the gold standard to study cardiac structure and function and is, therefore, key to evidence-based diagnostic and therapeutic decisions [24].

By assessing cardiovascular anatomy, myocardial structure and characterization, biventricular systolic function and flow, CMR can be used to study pathologies ranging from myocardial ischemia and viability to cardiomyopathies, as well as pericardial, valvular, congenital and vascular diseases [25].

A number of protocol sequences are currently used for imaging acquisition. These require ECG synchronization. Conventional sequences are acquired during cardiac cycles with short breath-holding periods.

Bright-blood cine sequences provide an excellent contrast between blood, myocardium and vessels, allowing the assessment of heart volumes and biventricular function, with CMR being a reference method for these calculations. Myocardial tissue structure characterization may be obtained using sequences such as black-blood imaging T1-weighted, T2-weighted (for edema) and fat-saturation. By administering gadolinium, and using an inversion-recovery sequence, it is possible to obtain different patterns of late gadolinium enhancement (LGE-CMR) of the diseased myocardium and characterize clinical conditions, like ischemic heart disease and cardiomyopathies. Using a saturation-recovery sequence, myocardial perfusion may be assessed during gadolinium infusion, after pharmacological stress. Other important applications include flow quantification (for valvular and vascular assessment) by phase-contrast sequences and MR angiography for vessel evaluation [25]. Recently, T1 and T2 mapping are available for myocardial characterization in diseased states, namely for diagnosing diffuse fibrosis, inflammation, cardiac amyloid, Fabry disease and iron overload [26].

In some stroke patients, CMR performance may be challenging, but it is feasible, in both young and older patients, as long as they are able to hold their breath for some seconds, stand still and understand the instructions for imaging acquisition. In exceptional cases, were further diagnosis is intended (e.g. tissue characterization) in non-cooperative patients, the use of sedation and of respiratory navigators, as needed, may ensure appropriate imaging. Although not routinely performed due to increased acquisition time, the use of respiratory navigators is feasible and available in most scanners.

[25]. Contraindications to CMR include claustrophobia, implanted ferromagnetic material like intraocular foreign bodies, cochlear implants, and usually non-conditional implantable electronic devices. Patients with severe renal failure with an eGFR below $30 \mathrm{~mL} / \mathrm{min} / 1.73 \mathrm{~m}^{2}$ should not receive gadolinium due to the risk of nephrogenic systemic fibrosis [25]. Patients in critical care units should undergo CMR if the diagnostic information is crucial. In this case, appropriate stabilization should be performed, with full monitoring during the exam, which may be achieved with respiratory and hemodynamic support with MR compatible equipment [27].

\section{Challenges of CMR in stroke assessment}

CMR is probably not feasible, in most patients, in the acute phase of stroke where other techniques like echocardiography or cardiac CTA have a more appropriate role for detecting cardiac thrombi. CMR has long acquisition times and requires patients to stand still. In addition, patients need to be instructed to hold their breath. This may be difficult in stroke patients, especially in those with severe deficits (who might have a higher chance of having a cardiac source of embolism). CMR is complex and needs experienced physicians and trained technicians. Although costs of CMR are variable in different countries, it is more costly than cardiac CTA. However, CMR has no radiation and provides a larger amount of information than cardiac CTA, namely on heart 
function, flow and myocardial characterization that may be crucial for decision-making, thus being a valid option when safely and appropriately used.

\section{CMR for the investigation of established causes of cardioembolic stroke}

Echocardiography is the first-line exam to study a cardioembolic etiology due to its availability and ability to be used at bedside. However, it is operator dependent and has acoustic window limitations and considerable measurement variability. CMR may be complementary and have an incremental value to echocardiography. CMR does not have window restrictions, has high image resolution and reproducibility and has a unique capability to characterize tissue.

Cardiac CTA was recently suggested as an alternative technique for the workup of stroke of undetermined etiology, due to its high sensitivity and specificity for thrombus detection [28] in comparison with TEE as a reference standard [29]. Although cardiac CTA is a promising alternative to echocardiography it uses radiation and requires iodinated contrast. Acquisition of cardiac CTA images could potentially be done in the acute stroke phase after the acquisition of brain CT. Delayed imaging may be diagnostic and needs a lower radiation dose. However, to ensure quality and diagnostic performance, ECG-gating and patient's breath-holding is advised. The presence of arrhythmias and tachycardia may be a limitation that advanced scanners in the future may overcome. A recent meta-analysis that compared cardiac CTA and TEE suggests a good accuracy to detect cardiac thrombi in ischemic stroke but advises caution since included studies were small, heterogeneous and in most studies both techniques were performed days apart from each other [30]. Also, cardiac CTA does not provide information concerning function or flow and is limited for tissue characterization.

CMR provides not only an accurate diagnosis of thrombi, flow velocities in the LAA and aorta atheroma characterization but also detects and characterizes tumors, myocarditis, cardiomyopathies, atrial cardiomyopathy and silent myocardial infarction which are potential sources of embolism (Table 1).

\section{Atrial septal defect and patent foramen ovale}

CMR is useful for the diagnosis of an atrial septal defect (ASD) when echocardiography is inconclusive and can help to quantify the size of the left-to-right shunt (Fig. 1). It is also useful for the diagnosis of a superior or inferior sinus venosus ASD, for multiple atrial septal defects and for the detection of associated anomalous pulmonary veins [31].

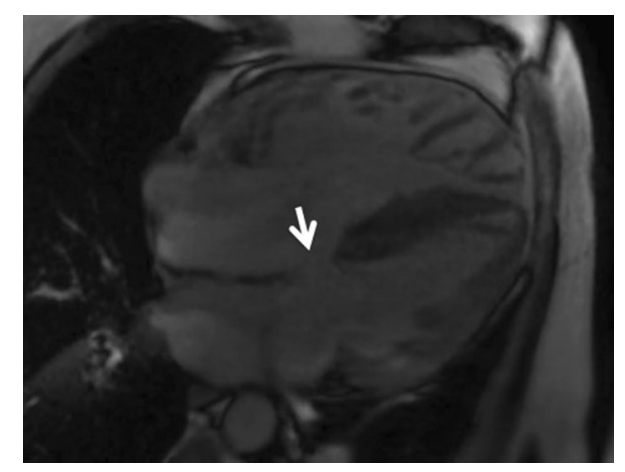

Fig. 1 Still image from a cine MR sequence in four-chamber plane depicting an "ostium primum" defect (arrow)
Table 1 Summary of current CMR role in stroke etiological investigation

\begin{tabular}{lllll}
\hline & TTE & TEE & CMR & Cardiac CTA \\
\hline Investigation of TOAST cardioembolic causes & & & \\
Atrial septal defect & $+/+++$ & +++ & +++ & +++ \\
PFO & + & +++ & + & $+/++$ \\
LV thrombus & + & $++/+++$ & +++ & +++ \\
LAA thrombus & - & +++ & +++ & +++ \\
Cardiac tumors & + & ++ & $+++^{\mathrm{a}}$ & +++ \\
Endocarditis & ++ & +++ & $-/+$ & ++ \\
Aortic atheroma & - & $++/+$ & $++/+++^{\mathrm{a}}$ & +++ \\
New etiologic mechanisms & & & & \\
Atrial cardiomyopathy & - & $+/++$ & $+++^{\mathrm{a}}$ & + \\
Silent myocardial infarction & + & + & $+++^{\mathrm{a}}$ & ++ \\
Myocarditis and cardiomyopathy & $+/+++$ & $+/++$ & $+++^{\mathrm{a}}$ & $-/++{ }^{\mathrm{b}}$ \\
\hline
\end{tabular}

TTE transthoracic echocardiogram, TEE transesophageal echocardiogram

${ }^{a}$ Additional tissue characterization

${ }^{\mathrm{b}}$ Anatomic features 
TEE often needing the performance of a Valsalva Maneuver is the modality of choice for the diagnosis of PFO due to its high spatial and temporal resolution [32].

PFO may be detected with transthoracic echocardiogram (TTE) and agitated saline contrast but its sensitivity is lower than TEE, as it depends on the acoustic window and does not provide the anatomic characteristics of the left atrium or the quantification of microbubbles [33].

Detecting a PFO is challenging for CMR due to its usual small size and because frequently the right-to-left shunt develops after a Valsalva maneuver that occurs during few cardiac cycles. Since CMR cine acquisition needs several cycles' for imaging, this may preclude the detection of turbulence from the shunt. A first-pass perfusion CMR method with the evaluation of enhancement curves in the left and right atria has been able to successfully diagnose PFO but this technique needs to be validated [34]. The presence of a septal aneurysm, defined as septal bowing $>10 \mathrm{~mm}$, is easily detected by cine CMR [35].

\section{Cardiac thrombi}

Thrombi are the most frequent cardiac masses. They tend to occur in areas of stasis, such as the left atrium and the left atrial appendage in association to atrial dilation and AF, or in the left ventricle in the presence of regional wall motion abnormalities or global ventricular dysfunction (Fig. 2).

Thrombi are diagnosed using T1- and T2-weighted blackblood sequences, cine CMR and particularly by the absence of early or late gadolinium enhancement since they are avascular which allows for them to be diagnosed against a differential diagnosis of other masses [36]. CMR is more accurate than echocardiography in detecting thrombi [37] and should be considered when echocardiography is inconclusive.

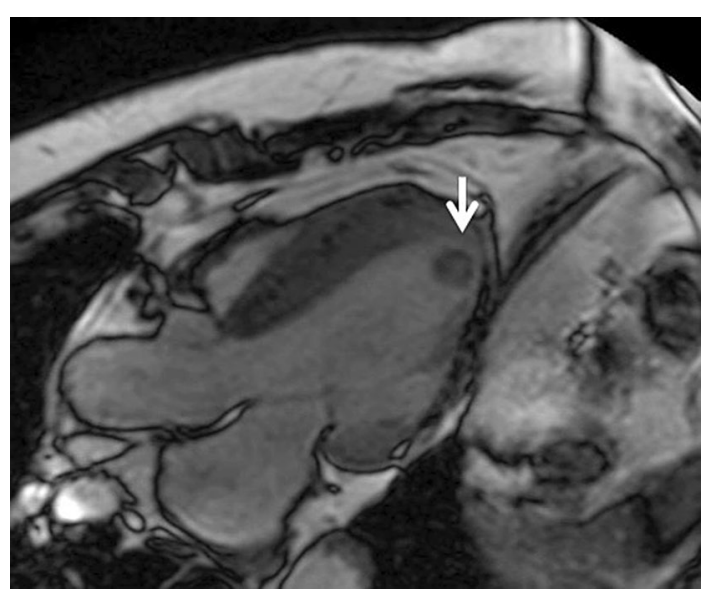

Fig. 2 Still image from a cine MR sequence in three-chamber plane showing a thrombus in the apex (arrow)
Baher et al. [38] conducted a prospective study of 106 patients with ischemic stroke and transient ischemic attacks. After routine work-up, in $21 \%$ of patients CMR allowed a reclassification of cryptogenic causes into cardioembolic, mainly by improving the diagnosis of thrombi.

LV thrombi detected by LGE CMR but not by echocardiography are associated with a similar risk of embolism compared to that detected by both LGE CMR and echocardiography [39].

\section{Tumors}

Tumors can embolize, be arrhythmogenic or cause heart failure. CMR is a more valuable technique to diagnose cardiac tumors than echocardiography [40], due to its high tissue contrast, versatility in image planes, unique value for tissue characterization, namely for water and fat identification, which have specific patterns on T1 and T2-weighted techniques. Contrast-enhanced CMR can also evaluate tumor vascularity and fibrosis. Due to its large field of view, CMR can be used to detail the tumor's relationship to other structures.

The majority of primary cardiac tumors are benign and myxomas are the most frequent type [41]. They are typically attached to the atrial septum, although they may occur in any cavity (Fig. 3a). They are often isointense on T1, hyperintense on T2 and heterogeneous after contrast. Myxomas have a high embolization risk and surgical removal is indicated. Papillary fibroelastomas are small benign endocardial papillomas affecting predominantly the cardiac valves (Fig. 3b). They are small and highly mobile masses, homogeneous and hypointense on cine images, isointense on $\mathrm{T} 1$ and hyperintense on T2, and heterogeneous with contrast enhancement allowing for their differential diagnosis from vegetations. Clinically, fibroelastomas can be asymptomatic or associated with systemic embolization [41]. Lipomas are benign tumors that often arise from the epicardial surface. They are characterized by high intensity on $\mathrm{T} 1$ that is suppressed by fat saturation pulses. Other primary tumors like fibromas or rhabdomyomas are intramural and have no embolic potential.

Primary malignant tumors are rare, more frequently sarcomas or less often lymphomas, while metastatic tumors are $20-40 \times$ more frequent [41]. Malignant tumors may be intramural but often protrude into the cardiac cavities and may embolize. Characteristically, these tumors have irregular contours and are infiltrative, heterogeneous in signal and on contrast-enhancement.

\section{Infectious endocarditis}

Cardioembolic stroke occurs in up to $35 \%$ of patients with infective endocarditis [42]. Very mobile vegetations that 
Fig. 3 a Still image from a cine MR sequence in four-chamber plane showing a myxoma attached to the interatrial septum (arrow), b left ventricle short-axis T1W image showing a fibroelastoma attached to the mitral valve (arrow)

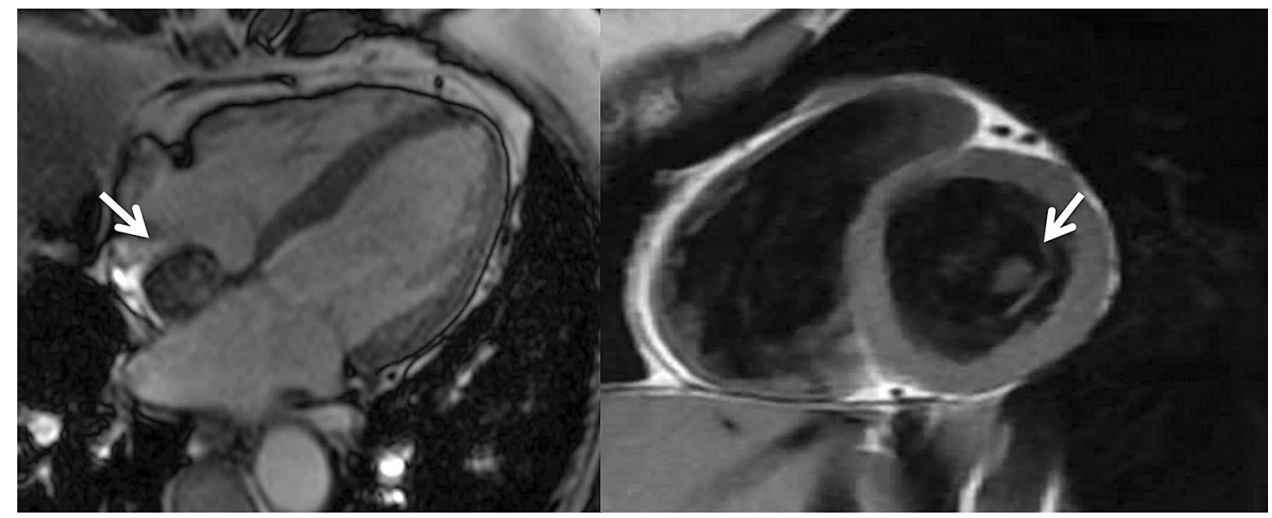

are $>10 \mathrm{~mm}$ have a higher embolization risk [43]. CMR is not a first-line method for detecting valvular vegetations because it has limited spatial resolution for small mobile structures. Transesphageal echocardiography is the reference method for the diagnosis of infective endocarditis, complemented by cardiac CT and 18F-FDG PET-CT [43] in some cases.

CMR may be useful for diagnosing perivalvular complications in IE patients, such as perivalvular abscesses and inflammatory processes involving the myocardium [44]. Also, valve insufficiency severity and its hemodynamic significance can be assessed by CMR.

\section{Aortic atheroma and other conditions}

Atheroma plaques that are more than $4 \mathrm{~mm}$ in size, ulcerated atheroma plaques, and atheroma plaques with mobile components considered as complex lesions, are associated with a fourfold risk of thromboembolism in comparison with plaques without complex features [45].

TEE is the reference method to study aortic atheroma located in the arch and in the proximal descending aorta. Black-blood CMR sequences have shown good correlation with TEE [46]. CMR has the advantage of an unrestricted view field and the potential of diagnosing mural thrombi. High-resolution scanners have the potential to diagnose intraplaque hemorrhage and evaluate lipid content. However, calcification is not well detected by CMR. Contrastenhanced MR angiography provides images of protruding complex plaques with diagnostic power [47].

CMR has been used to evaluate carotid atherosclerosis. In a study using histological validation obtained from carotid endarterectomy specimens, CMR was able to characterize lipid-rich necrotic cores, fibrous caps, intraplaque haemorrhage, calcification, and inflammation [48].

\section{CMR for investigation of cryptogenic stroke}

CMR has helped in the last years to identify possible stroke causes that are not part of standard ischemic stroke etiological classifications. These situations include atrial cardiomyopathy, cardiomyopathies and silent myocardial infarctions.

\section{Atrial cardiomyopathy}

Recent studies suggest that AF is the final point of a disease characterized by morphological and structural changes in the atria. There is increasing evidence that a structural atrial condition defined as fibrotic atrial cardiomyopathy can precede the occurrence of AF [49]. These changes could increase the probability of thrombi formation. A decrease in left atrial ejection fraction could induce stasis, and an increase in atrial fibrosis could lead to both stasis and endocardium changes that favor thromboembolism [46]. CMR with late gadolinium enhancement can identify atrial fibrosis. Fonseca et al. compared the left atrium (LA) of patients with different ischemic stroke causes using CMR [50]. Patients with structural changes on echocardiography considered as causal for stroke in the TOAST classification were excluded. One hundred and eleven patients were evaluated. A 3Tesla CMR was performed. Patients with an undetermined cause had a higher percentage of LA fibrosis $(p=0.03)$ than patients with other stroke causes and lower, although not statistically significant, values of LA ejection fraction. Patients with atrial fibrillation and undetermined stroke causes showed a similar value of atrial fibrosis. Similar results were found by Tandon et al., in a study using CMR, odds ratios of ESUS per quartile of fibrosis were 3.17 (95\% CI 
1.05-9.52, $p=0.041, \mathrm{CHA}_{2}$ DVASc score adjusted) [51]. This LA phenotype that was found in patients with an undetermined stroke cause supports the hypothesis that fibrosis is in the causal pathway of cardioembolic stroke independently of AF [51]. Further studies are needed to determine the role of anticoagulation in primary and secondary stroke prevention in patients with high atrial fibrosis.

Women with AF have a higher risk of stroke than men who have AF. In a study using CMR, female sex was found to be independently associated with left atrial fibrosis, after controlling for confounders such as AF and age. More studies are needed to understand if this contributes to the increased stroke risk related to $\mathrm{AF}$ in women compared with men [52].

The MESA study aimed to assess the association of baseline left atrial phasic function measured with CMR in a population free of known cardiovascular disease and incident ischemic cerebrovascular events [53]. The authors found that reduced total left atrial emptying fraction was associated with incident ischemic cerebrovascular events independently of known cerebrovascular risk factors and incident AF. Assessment of LA function by CMR may, therefore, add further information to stratify asymptomatic individuals at risk for ischemic stroke.

\section{Silent myocardial infarction}

A recent study aimed to investigate if CMR could be useful in identifying unrecognized myocardial infarction (MI) and if it was associated with cerebral infarction [54]. It used data from a cross-sectional study of ICELAND MI. A community-dwelling cohort of Icelandic individuals aged 67-93 years who underwent brain MRI and LGE CMR were enrolled. Unrecognized MI was defined as CMR evidence of MI without a history of clinically evident MI. Cerebral infarctions on brain MRI were included regardless of associated symptoms. Among 925 participants, there were 221 participants (23.9\%) with CMR evidence of MI, of whom 153 had had an unrecognized MI. There were 308 participants $(33.3 \%)$ with brain MRI evidence of cerebral infarction; 93 (10.0\%) had embolic infarcts of undetermined source. After adjustment for demographic factors and vascular risk factors, the odds ratio (OR) of having cerebral infarction was 2.0 (95\% CI 1.2-3.4; $p=0.01$ ) for recognized MI and 1.5 (95\% CI 1.02-2.2; $p=0.04$ ) for unrecognized MI. Unrecognized MI was also associated with embolic infarcts of undetermined source (OR 2.0 [95\% CI 1.1-3.5]; $p=0.02$ ). These findings suggest that unrecognized MI may be a novel risk factor for cardiac embolism and cerebral infarction.

\section{Cardiomyopathies}

CMR has diagnostic utility in the assessment of cardiomyopathies. Cardiomyopathies are rare conditions defined by structural and functional abnormalities of the ventricular myocardium that are unexplained by flow-limiting coronary artery disease or abnormal loading conditions. A recent study was performed to study if CMR could be useful in identifying previously undiagnosed cardiomyopathies in a cohort of patients with ischemic stroke who had undergone unremarkable standard etiological investigation [55]. One hundred thirty-two patients with a mean age of 68.4 years were included. In seven patients $(5.3 \%$, 95\% IC 2.59-10.54\%), CMR identified a cardiomyopathy. Four patients had hypertrophic cardiomyopathy (HCM), two had restrictive cardiomyopathy, and one had noncompaction cardiomyopathy. Six of these patients had been classified as having undetermined stroke and one patient as having cardioembolic stroke (AF). A higher frequency of HCM was found in the entire cohort and in the undetermined cause group compared to the general population (3.03\% and 5.81\% vs $0.2 \%$, respectively, $p=0.001$ and $p<0.001)$. The frequency of noncompaction cardiomyopathy was also higher in the cohort ( $0.76 \%$ vs $0.05 \%$, respectively, $p<0.001)$.

CMR LGE extent ( $>14.4 \%$ ) has been found to be an independent predictor for thromboembolic complications in patients with HCM [56].

Another study using CMR, evaluated 30 young cryptogenic stroke patients and 30 controls to evaluate if noncompaction cardiomyopathy could be a possible stroke cause [57]. In a multivariate conditional logistic regression model, the percentage of non-compacted LV volume (odds ratio [OR] 1.55, 95\% confidence interval [CI] 1.10-2.18, $p=0.011$ ) was found to be independently associated with cryptogenic ischemic stroke.

\section{Conclusions and future directions}

CMR may be useful to clarify established cardioembolic stroke causes and to evaluate possible novel mechanisms of ischemic stroke (Table 1). Its use should be considered to evaluate ischemic stroke patients that are classified as having an undetermined stroke etiology after a standard evaluation. Atrial fibrosis may play an important role in stroke pathophysiology. CMR could be useful to phenotype patients with atrial fibrosis in clinical trials. Such studies would be useful to clarify the best secondary prevention strategies for these patients.

In the future, new techniques may simplify and shorten the CMR protocol increasing its use and making it more widely available. However, because it is not viable in a global health care perspective to perform advanced imaging 
in all patients, the identification of specific patterns (demographics or imaging) could help to select which patients need a more thorough investigation with CMR.

\section{Compliance with ethical standards}

Conflicts of interest Nothing to disclose.

\section{References}

1. Adams HP Jr, Bendixen BH, Kappelle LJ et al (1993) Classification of subtype of acute ischemic stroke. Definitions for use in a multicenter clinical trial TOAST. Trial of Org 10172 in acute stroke treatment. Stroke 24(1):35-41. https://doi.org/10.1161/01. str.24.1.35

2. Amarenco P, Bogousslavsky J, Caplan LR, Donnan GA, Wolf ME, Hennerici MG (2013) The ASCOD phenotyping of ischemic stroke (Updated ASCO Phenotyping). Cerebrovasc Dis 36:1-5. https://doi.org/10.1159/000352050

3. Shimizu T, Kashima S, Akiyama H, Isahaya K, Hasegawa Y (2020) The ASCOD phenotyping of embolic strokes of undetermined source. J Stroke Cerebrovasc Dis 29(2):104491. https://doi. org/10.1016/j.jstrokecerebrovasdis.2019.104491

4. McMahon NE, Bangee M, Benedetto V et al (2020) Etiologic workup in cases of cryptogenic stroke a systematic review of international clinical practice guidelines. Stroke 51:1419-1427. https://doi.org/10.1161/strokeaha.119.027123

5. Hart RG, Diener HC, Coutts SB et al (2014) Embolic strokes of undetermined source: the case for a new clinical construct. Lancet Neurol 13(4):429-438. https://doi.org/10.1016/S1474 -4422(13)70310-7ESUSHartetal,2014

6. Kim SJ, Allen JW, Bouslama M et al (2017) Carotid webs in cryptogenic ischemic strokes: a matched case-control study. J Stroke Cerebrovasc Dis 28(12):104402. https://doi.org/10.1016/j.jstro kecerebrovasdis.2019.104402

7. Coutinho JM, Derkatch S, Alphonse RJ, Potvin ARJ et al (2017) Carotid artery web and ischemic stroke: a case-control study. Neurology 88(1):65-69. https://doi.org/10.1212/WNL.0000000000 003464

8. Zhang AJ, Dhruv P, Choi P et al (2018) A systematic literature review of patients with carotid web and acute ischemic stroke. Stroke 49(12):2872-2876. https://doi.org/10.1161/strok eaha.118.021907

9. Ntaios G, Pearce LA, Veltkamp R et al (2020) Potential embolic sources and outcomes in embolic stroke of undetermined source in the NAVIGATE-ESUS trial. Stroke 51(6):1797-1804. https:// doi.org/10.1161/STROKEAHA.119.028669

10. Martinez-Majander RN, Ntaios G, Liu YY et al (2020) Rivaroxaban versus aspirin for secondary prevention of ischaemic stroke in patients with cancer: a subgroup analysis of the NAVIGATE ESUS randomized trial. Eur J Neurol. 27(5):841-848. https://doi. org/10.1111/ene. 14172

11. Ntaios G, Pearce LA, Meseguer E et al (2020) Aortic arch atherosclerosis in patients with embolic stroke of undetermined source: an exploratory analysis of the NAVIGATE ESUS trial. Stroke 50(11):3184-3190. https://doi.org/10.1161/STROK EAHA.119.025813

12. Ameriso SF, Amarenco P, Pearce LA et al (2020) Intracranial and systemic atherosclerosis in the NAVIGATE ESUS trial: recurrent stroke risk and response to antithrombotic therapy. J Stroke Cerebrovasc Dis 29(8):104936. https://doi.org/10.1016/j.jstrokecer ebrovasdis.2020.104936
13. Uchiyama S, Toyoda K, Kitagawa K et al (2019) Branch atheromatous disease diagnosed as embolic stroke of undetermined source: a sub-analysis of NAVIGATE ESUS. Int J Stroke 14(9):915-922. https://doi.org/10.1177/1747493019852177

14. Kamel H, Pearce LA, Ntaios G et al (2020) Atrial cardiopathy and nonstenosing large artery plaque in patients with embolic stroke of undetermined source. Stroke 51(3):938-943. https://doi. org/10.1161/STROKEAHA.119.028154 (PMID: 31893985)

15. Ntaios G, Perlepe K, Sirimarco G et al (2019) Carotid plaques and detection of atrial fibrillation in embolic stroke of undetermined source. Neurology 92(23):e2644-e2652. https://doi.org/10.1212/ WNL.0000000000007611

16. Porambo ME, DeMarco JK (2020) MR imaging of vulnerable carotid plaque. Cardiovasc Diagn Ther. 10(4):1019-1031. https ://doi.org/10.21037/cdt.2020.03.12

17. Mark IT, Nasr DM, Huston J et al (2020) Embolic stroke of undetermined source and carotid intraplaque hemorrhage on MRI: a systemic review and meta-analysis. Clin Neuroradiol. https://doi. org/10.1007/s00062-020-00921-2

18. Ospel JM, Singh N, Marko M et al (2020) Prevalence of ipsilateral nonstenotic carotid plaques on computed tomography angiography in embolic stroke of undetermined source. Stroke 51(6): 17431749. https://doi.org/10.1161/STROKEAHA.120.029404

19. Siegler JE, Thon JM, Woo JH, Do D, Messé SR, Cucchiara B (2020) Prevalence of nonstenotic carotid plaque in stroke due to atrial fibrillation compared to embolic stroke of undetermined source. J Stroke Cerebrovasc Dis 28(10):104289. https://doi. org/10.1016/j.jstrokecerebrovasdis.2019.07.005

20. Mac Grory B, Emmer BJ, Roosendaal SD, Zagzag D, Yaghi S, Nossek E (2020) Carotid web: an occult mechanism of embolic stroke. J Neurol Neurosurg Psychiatry. https://doi.org/10.1136/ jnnp-2020-323938

21. Kim SJ, Allen JW, Bouslama M et al (2019) Carotid webs in cryptogenic ischemic strokes: a matched case-control study. J Stroke Cerebrovasc Dis 28(12):104402. https://doi.org/10.1016/j.jstro kecerebrovasdis.2019.104402

22. Fonseca AC, Ferro JM (2015) Cryptogenic stroke. Eur J Neurol 22:618-623

23. Fonseca AC, Brito D, Pinho-e-Melo T, Geraldes R, Canhão P, Caplan LR, Ferro JM (2014) N-terminal pro-brain natriuretic peptide shows diagnostic accuracy for detecting atrial fibrillation in cryptogenic stroke patients. Int J Stroke 9:419-425

24. Childs H, Ma L, Ma M et al (2011) Comparison of long and short axis quantification of left ventricular volume parameters by cardiovascular magnetic resonance, with ex-vivo validation. J Cardiovasc Magn Reson 13:40

25. Hundley WG, Bluemke DA, Finn JP et al (2010) ACCF/ACR/ AHA/NASCI/SCMR 2010 expert consensus document on cardiovascular magnetic resonance: a report of the American College of Cardiology Foundation Task Force on Expert Consensus Documents. J Am Coll Cardiol 55:2614-2662

26. Messroghli DR, Moon JC, Ferreira VM et al (2017) Clinical recommendations for cardiovascular magnetic resonance mapping of $\mathrm{T} 1, \mathrm{~T} 2, \mathrm{~T} 2 *$ and extracellular volume: a consensus statement by the Society for Cardiovascular Magnetic Resonance (SCMR) endorsed by the European Association for Cardiovascular Imaging (EACVI). J Cardiovasc Magn Reson 19:75

27. Bonnefoy-Cudraz E, Bueno H, Casella G, De Maria E, Fitzsimons D, Halvorsen S, Hassager C, Iakobishvili Z, Magdy A, Marandi T, Mimoso J, Parkhomenko A, Price S, Rokyta R, Roubille F, Serpytis P, Shimony A, Stepinska J, Tint D, Trendafilova E, Tubaro M, Vrints C, Walker D, Zahger D, Zima E, Zukermann R, Lettino M (2018) Editor's choice-acute cardiovascular care association position paper on intensive cardiovascular care units: an update on their definition, structure, organisation and function. Eur Heart J Acute Cardiovasc Care 7(1):80-95 
28. Romero J, Husain SA, Kelesidis I, Sanz J, Medina HM, Garcia MJ (2016) Detection of left atrial appendage thrombus by cardiac computed tomography in patients with atrial fibrillation: a metaanalysis. Circ Cardiovasc Imaging 6:185-194

29. Manning WJ, Weintraub RM, Waksmonski CA et al (1995) Accuracy of transesophageal echocardiography for identifying left atrial thrombi. A prospective, intraoperative study. Ann Intern Med 123:817-822

30. Groenevel I, Guglielmi V, Leeflang M et al (2020) CT angiography vs echocardiography for detection of cardiac thrombi in ischemic stroke: a systematic review and meta-analysis. J Neurol 267:1793-1801

31. Babu-Narayan SV, Giannakoulas G, Valente AM, Li W, Gatzoulis MA (2016) Imaging of congenital heart disease in adults. Eur Heart J 37:1182-1195

32. Miranda B, Fonseca AC, Ferro JM (2018) Patent foramen ovale and stroke. J Neurol 265(8):1943-1949

33. Silvestry FE, Cohen MS, Armsby LB et al (2015) Guidelines for the echocardiographic assessment of atrial septal defect and patent foramen ovale: from the American Society of Echocardiography and Society for Cardiac Angiography and Interventions. J Am Soc Echocardiogr 28(8):910-958

34. Mohrs OK, Petersen SE, Erkapic D et al (2005) Diagnosis of patent foramen ovale using contrast-enhanced dynamic MRI: a pilot study. AJR Am J Roentgenol 84:234

35. Fernandez RS, Diaz CM, Garcia ER, Calvo AM, Pan AR et al (2011) Atrial abnormalities: spectrum on MRI. AJR Am J Roentgenol 197:W635-W642

36. Weinsaft JW, Kim HW, Shah DJ et al (2008) Detection of left ventricular thrombus by delayed-enhancement cardiovascular magnetic resonance prevalence and markers in patients with systolic dysfunction. J Am Coll Cardiol 52:148-157

37. Srichai MB, Junor C, Rodriguez LL et al (2006) Clinical, imaging, and pathological characteristics of left ventricular thrombus: a comparison of contrast-enhanced magnetic resonance imaging, transthoracic echocardiography, and transesophageal echocardiography with surgical or pathological validation. Am Heart J 152:75-84

38. Baher A, Mowla A, Kodali S et al (2014) Cardiac MRI improves identification of etiology of acute ischemic stroke. Cerebrovasc Dis 37:277-284

39. Velangi PS, Choo C, Chen KA, Kazmirczak F, Nijjar PS, Farzaneh-Far A, Okasha O, Akçakaya M, Weinsaft JW, Shenoy C (2019) Long-term embolic outcomes after detection of left ventricular thrombus by late gadolinium enhancement cardiovascular magnetic resonance imaging: a matched cohort study. Circ Cardiovasc Imaging 12:e009723

40. Itbach MI, Squire SW, Kudithipudi V, Castellano L, Sorrell VL, (2007) Cardiac MRI is complementary to echocardiography in the assessment of cardiac masses. Echocardiography 24:286-300

41. Rosário M, Fonseca AC, Sotero FD, Ferro JM (2019) Neurological complications of cardiac tumors. Curr Neurol Neurosci Rep 19:15

42. Sotero FD, Rosário M, Fonseca AC, Ferro JM (2019) Neurological complications of infective endocarditis. Curr Neurol Neurosci Rep 19:23
43. Habib G, Lancellotti P, Antunes MJ et al (2015) 2015 ESC guidelines for the management of infective endocarditis. Eur Heart J 36:3075-3128

44. Dursun M, Yilmaz S, Y1lmaz E et al (2015) The utility of cardiac MRI in diagnosis of infective endocarditis: preliminary results. Diagn Interv Radiol 21:28-33

45. Meissner I, Khandheria BK, Sheps SG et al (2004) Atherosclerosis of the aorta: risk factor, risk marker, or innocent bystander? A prospective population-based transesophageal echocardiography study. J Am Coll Cardiol 44:1018-1024

46. Faber T, Rippy A, Hyslop WB, Hinderliter A, Sen S (2013) Cardiovascular MRI in detection and measurement of aortic atheroma in stroke/TIA patients. J Neurol Disord 1:139

47. Corti R, Fuster V (2011) Imaging of atherosclerosis: magnetic resonance imaging. Eur Heart J 32:1709-1719

48. Kerwin WS, Miller Z, Yuan C (2017) Imaging of the high-risk carotid plaque: magnetic resonance imaging. Semin Vasc Surg 30:54-61

49. Kottkamp H (2013) Human atrial fibrillation substrate: towards a specific fibrotic atrial cardiomyopathy. Eur Heart J 34:2731-2738

50. Fonseca AC, Alves P, Inácio N, Marto JP, Viana-Baptista M, Pinho-E-Melo T, Ferro JM, Almeida AG (2018) Patients with undetermined stroke have increased atrial fibrosis: a cardiac magnetic resonance imaging study. Stroke 49(3):734-737

51. Tandon K, Tirschwell D, Longstreth WT Jr, Smith B, Akoum N (2019) Embolic stroke of undetermined source correlates to atrial fibrosis without atrial fibrillation. Neurology 93(4):e381-e387

52. Fonseca AC, Marto JP, Alves PN, Inácio N, Viana-Baptista M, Pinho E, Melo T, Ferro JM, Almeida AG (2018) Women who have ischemic strokes have a higher burden of left atrial fibrosis than men. Stroke 49:2584-2589

53. Habibi M, Zareian M, Ambale Venkatesh B, Samiei S, Imai M, Wu C, Launer LJ, Shea S, Gottesman RF, Heckbert SR, Bluemke DA, Lima JAC (2019) Left atrial mechanical function and incident ischemic cerebrovascular events independent of AF: insights from the MESA study. JACC Cardiovasc Imaging 12:2417-2427

54. Merkler AE, Sigurdsson S, Eiriksdottir G et al (2019) Association between unrecognized myocardial infarction and cerebral infarction on magnetic resonance imaging. JAMA Neurol 76:956-961

55. Fonseca AC, Marto JP, Pimenta D, Guimarães T, Alves PN, Inácio N, Viana-Baptista M, Pinho-E-Melo T, Pinto FJ, Ferro JM, Almeida AG (2020) Undetermined stroke genesis and hidden cardiomyopathies determined by cardiac magnetic resonance. Neurology 94:e107-e113

56. Hohneck A, Overhoff D, Doesch C, Sandberg R, Rudic B, Tueluemen E, Budjan J, Szabo K, Borggrefe M, Papavassiliu T (2020) Extent of late gadolinium enhancement predicts thromboembolic events in patients with hypertrophic cardiomyopathy. Circ J 84:754-762

57. Pöyhönen P, Kuusisto J, Järvinen V, Pirinen J, Räty H, Lehmonen L, Paakkanen R, Martinez-Majander N, Putaala J, Sinisalo J (2020) Left ventricular non-compaction as a potential source for cryptogenic ischemic stroke in the young: a case-control study. PLoS ONE 15(8):e0237228 